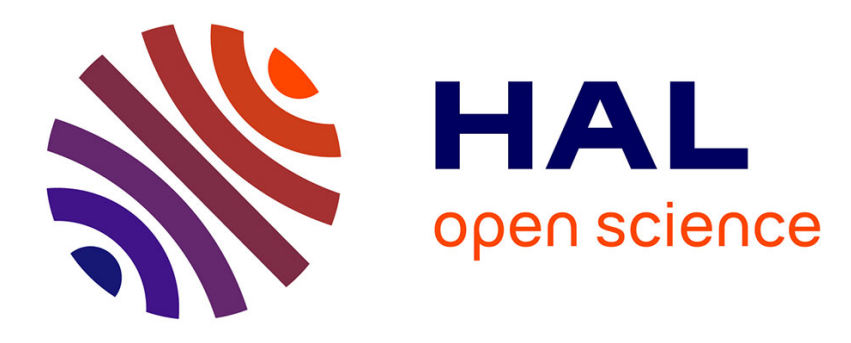

\title{
Le livre en héritage: les religieux mendiants face aux manuscrits médiévaux au XVIIe siècle
}

Fabienne Henryot

\section{To cite this version:}

Fabienne Henryot. Le livre en héritage: les religieux mendiants face aux manuscrits médiévaux au XVIIe siècle. Entre stabilité et itinérance. Livres et culture des ordres mendiants, Nov 2010, Paris, France. pp.439-458. hal-01103758

\section{HAL Id: hal-01103758 \\ https://hal.science/hal-01103758}

Submitted on 15 Jan 2015

HAL is a multi-disciplinary open access archive for the deposit and dissemination of scientific research documents, whether they are published or not. The documents may come from teaching and research institutions in France or abroad, or from public or private research centers.
L'archive ouverte pluridisciplinaire HAL, est destinée au dépôt et à la diffusion de documents scientifiques de niveau recherche, publiés ou non, émanant des établissements d'enseignement et de recherche français ou étrangers, des laboratoires publics ou privés. 


\section{Le livre en héritage : les religieux mendiants face aux manuscrits médiévaux au XVII'e siècle}

Publié dans : N. Bériou, M. Morard, D. Nebbiai (dir.), Entre stabilité et itinérance. Livres et culture des ordres mendiants, actes du colloque de Paris, 19-20 novembre 2010, Turnhout, Brepols, 2014, p. 439-458.

Fabienne Henryot Bibliothèque cantonale et universitaire de Lausanne LARHRA - UMR 5190 - Équipe RESEA

En 1644, le carme Louis Jacob publie à Paris son Traicté des plus belles bibliothèques publiques et particulières dans lequel il décrit, province après province, les plus prestigieuses collections de livres de son temps à travers le monde ${ }^{1}$. L'ouvrage, écrit sur une suggestion de Gabriel Naudé, est en fait une compilation d'observations cueillies dans des «topographies » précédemment publiées par d'autres, comme Juste Lipse ${ }^{2}$, Onuphre Panuin ${ }^{3}$ ou Ange Rocca ${ }^{4}$, deux religieux augustins. Ou encore, de témoignages aimablement envoyés par de serviables correspondants. Louis Jacob s'en est donc essentiellement remis à l'appréciation d'autrui, mais c'est lui qui a défini les critères qui déterminent une « belle » bibliothèque. D’abord, la quantité numérique : il ne mentionne que les collections dépassant les trois mille volumes, et si possible, quatre mille. Ensuite, la présence de manuscrits est indispensable pour donner de la valeur à une bibliothèque. Enfin, la conservation de livres issus d'un don prestigieux la rehausse encore.

Manuscrits et conservation au long cours sont donc deux éléments essentiels pour définir la bibliothèque aux yeux d'un religieux mendiant du milieu du XVIIe siècle. Pourtant, cette conjonction ne va pas de soi. En effet, l'univers mendiant est au début de l'époque moderne extrêmement hétérogène, et jusque dans le statut du livre et de l'étude que chaque ordre impose à ses membres. Les ordres anciens, dominicains ${ }^{5}$, cordeliers $^{6}$, carmes $^{7}$, augustins ${ }^{8}$, ont été réformés au tournant des XVIe et XVII e siècle, tandis que des branches nouvelles issues de ces familles religieuses, capucins, minimes et carmes déchaussés, s'implantent massivement dans le paysage clérical ${ }^{9}$.

\footnotetext{
${ }^{1}$ L. JACOB, Traicté sur les plus belles bibliothèques publiques et particulières qui ont esté et qui sont encore dans le monde, Paris, 1644.

2 J. LIPSE, De bibliothecis syntagma, Anvers, 1602.

3 O. PANUINIUS, Reipublicae Romanae commentariorum libri tres, Francfort, 1597.

4 A. ROCCA, Bibliotheca Angelica, litteratorum litterarumque amatorum commoditati dicata, Rome, 1608.

${ }^{5}$ B. MONTAGNES, Sébastien Michaelis et la réforme d'Occitanie (1594-1647), Rome, 1984.

${ }^{6} \mathrm{~F}$. MEYER, L. VIALLET (dir.), Identités franciscaines à l'âge des réformes, Clermont-Ferrand, 2005.

7 S.-M. BOUCHEREAUX, La réforme des carmes en France et Jean de saint Samson, Paris, 1950.

${ }^{8}$ Y. EELCHO, "The Communauté de Bourges. A reform-movement in France in the 16th and the 17th centuries", Analecta Augustianiana, 52(1989), p. 165-190.

9 B. DOMPNIER, «Les étapes de l'expansion des capucins en Europe à l'époque moderne (1600-1750) », M. Dmitriev, P. Gonneau, J.-L. Lemaître (dir.), Moines et monastères dans les sociétés de rite grec et latin, Genève, 1996, p. 139-155.
} 
Ces ordres connaissent évidemment un rapport au livre très différent. Les couvents d'ordres anciens, qui ont déjà un à trois siècles d'existence, ont une véritable tradition du livre. Les autres, contemporains de la prolifération de l'imprimé, rejoignent une réflexion plus récente sur la culture écrite et sa place dans la vie régulière, l'apostolat et la prédication ${ }^{10}$. Toutefois, il serait vain d'opposer de manière schématique ces deux groupes d'ordres anciens et nouveaux. Car les premiers ont également dû composer avec l'avènement massif du livre imprimé et renouveler leur approche de l'écrit et de la lecture à la fin du XVIe siècle ; et d'autre part, les ordres nouveaux ont tiré parti de la circulation des manuscrits médiévaux pour se pourvoir en documents anciens.

Le XVII siècle est pour les bibliothèques conventuelles un temps de naissance ou de renaissance. Même si elles nous sont moins bien connues que pour le siècle suivant, qui a laissé une grande abondance d'archives et de catalogues, on sait que le début du siècle est un temps d'accroissement substantiel, par le biais d'achats et de dons massifs, avant les difficultés causées par les troubles politiques et militaires ; le dernier tiers du siècle voit à nouveau les collections se reconstituer, autour de la notion réaffirmée de bien commun et utile à tous, et sous la surveillance attentive des provinciaux et des visiteurs qui scrutent annuellement les livres pour en vérifier l'orthodoxie et la pertinence dans une bibliothèque de pauvres clercs.

Mais cet essor profite surtout, voire exclusivement, aux imprimés. Le manuscrit n'a plus qu'un rôle secondaire dans les bibliothèques conventuelles. Son statut change dans l'organisation intellectuelle et topographique des collections, organisation en plein bouleversement à la suite de l'augmentation significative du volume des bibliothèques. Il convient donc de s'interroger sur la fonction du manuscrit ancien dans les bibliothèques des couvents mendiants et sur la capacité des religieux à en saisir le caractère sinon précieux et unique, du moins la nature profondément différente des livres imprimés qui se sont imposés dans les collections.

\section{La mesure d'un phénomène}

Il est possible de mesurer la place du manuscrit dans les bibliothèques en interrogeant le discours interne tenu sur les collections conventuelles, qui fait état du regard, fatigué ou admiratif, que les religieux posent sur leurs manuscrits et quelle place ils leur assignent dans les bibliothèques. Les grandes fresques brossées par les historiens, franciscains notamment, évoquent au détour des récits de fondation puis d'essor des couvents, les bibliothèques conventuelles. Ce genre de propos reste toutefois assez rare, et quand il existe, il n'est jamais ni détaillé ni explicite, signe d'un intérêt faible pour les bibliothèques en général et les manuscrits en particulier. Du reste, ce désintérêt est caractéristique de l'ensemble du monde régulier, au début du XVIIe siècle du moins : bénédictins, prémontrés ou cisterciens ne font pas davantage état de leur richesse en livres ${ }^{11}$.

Ainsi, en 1587, François de Gonzague publie son De origine seraphicae religionis, recueil de notices sur chaque couvent de frères mineurs, notices

\footnotetext{
${ }^{10} \mathrm{~F}$. HENRYOT, Livres et lecteurs dans les couvents mendiants (Lorraine, XVIe-XVIIIe siècles), Genève, 2013.

11 Par exemple, G. JONGELINCK, dans ses Notitiae abbatiarum ordinis cisterciensis per universum orbem liber primus continens monasteria regni Galliae, ducatum Britanniae ac Lotharingiae, Cologne, 1640, n'évoque pas les bibliothèques.
} 
malheureusement limitées à des considérations générales ${ }^{12}$. Pour la province de France parisienne, qui compte alors 22 couvents d'hommes, 4 seulement ont droit à une appréciation sur leur bibliothèque: celui de Rouen, dont F. de Gonzague vante l'architecture et la distribution des officines conventuelles, dont la bibliothèque ${ }^{13}$; celui de Toul en Lorraine, qui dispose d'une «instructissima bibliotheca » ${ }^{14}$; celui de SaintLouis de Valognes, dont la bibliothèque «est abondamment pourvue des meilleurs livres ${ }^{15}$; enfin celui de Château-Thierry, qui possède « une bibliothèque incomparable, renfermant les meilleurs et les plus nombreux livres » ${ }^{16}$. Aucune distinction n'est faite entre imprimés et manuscrits, et on dispose encore moins de détails sur tel ou tel ouvrage rare ou précieux. Des bibliothèques connues alors pour leur richesse sont même passées sous silence, comme celle du couvent de Nancy. Dans les autres provinces, les silences sont également plus nombreux que les propos explicites. Pour la province de France, seul le couvent de Reims, pour un territoire qui compte quarante couvents, est assorti d'une précision : F. de Gonzague explique que la bibliothèque et le cloître ont été construits grâce aux libéralités d'Albéric, chanoine de la cathédrale ${ }^{17}$. Enfin, aucun des cinquante couvents de la province Saint-Bonaventure ne comporte de remarque sur la bibliothèque. Finalement, l'ouvrage de F. de Gonzague nous informe surtout sur l'appréciation - en l'occurrence, l'indifférence - d'un religieux sur l'état des collections de livres de son ordre.

En 1619, son confrère Jacques Fodéré prend à son tour la plume pour relater, dans l'ordre chronologique des fondations conventuelles, l'histoire des couvents qui composent l'ancienne province de "Bourgogne ${ }^{18}$, en réalité un vaste territoire qui s'étend, au nord des confins de la Lorraine, au sud, au pays de Vaud et à l'arc lémanique, en passant par l'Auvergne et la Franche-Comté. L'auteur a été lui-même provincial de la province Saint-Bonaventure (1606-1609) et à ce titre, il a visité l'ensemble des couvents de sa province. Prédicateur, historien, il est un homme de livres et d'érudition ${ }^{19}$. Son regard, qu'on suppose averti, sur les collections conventuelles peut donc nous guider. Il décrit 44 couvents de cordeliers de l'observance ${ }^{20}$ et treize d'entre eux donnent lieu à un commentaire sur la bibliothèque. À nouveau, les silences sont donc importants; mais la nature de ces commentaires est plus variée. On doit distinguer deux types de remarques : celles qui accordent à la bibliothèque une importante fonction mémorielle, et celles qui en font un objet historique.

D'abord, les appréciations positives des fonds. Ces remarques louangeuses sont toujours associées à un individu, gardien érudit ou consciencieux qui a eu à cœur d'améliorer la bibliothèque. L'examen de celle-ci par Fodéré est donc d'abord une affaire de mémoire. Ainsi, le franciscain attire l'attention sur Guillaume Marico, gardien et prédicateur à la fin du xve siècle, "qui a peuplé la Bibliotheque de sondict convent de Rion, d'un grand nombre de beaux et bons livres » ${ }^{21}$ sans préciser s'il s'agit de

\footnotetext{
12 François de Gonzague, De origine seraphicae religionis franciscanae ejusque progressibus, de regularis observantiae institutione..., Rome, 1587.

13 Ibid., p. 583.

${ }^{14}$ Ibid., p. 584.

15 Ibid., p. 587.

16 Ibid., p. 589.

17 Ibid., p. 555-556.

18 J. FODÉRÉ, Narration historique et topographique des convens de l'ordre S. François, et monastères S. Claire, erigez en la province anciennement appellée de Bourgongne, à présent de S. Bonaventure..., Lyon, 1619.

${ }^{19}$ L. WADDING, Scriptores ordinis minorum, rééd. Rome, 1806, p. 124.

20 Ces couvents se répartissent ainsi : custodie de Lyon (7); custodie de Bourgogne (10) ; custodie d'Auvergne (11) ; custodie de Franche-Comté (7) ; custodie du Dauphiné (3) ; custodie de Savoie (6).

${ }^{21}$ J. FODÉRÉ, Narration historique..., p. 333.
} 
manuscrits ou d'incunables. Aux Thons, fondation franciscaine de la fin du XVe siècle à la limite méridionale du diocèse de Toul, «l'une des singularitez remarquables de ce Convent est, qu'il y a une des plus belles Bibliotheques, bien peuplee de livres, que fut lors en la Province; vray est que ce sont tous de livres anciens $»^{22}$. Toutefois, ces remarques s'appliquent surtout à des personnages contemporains, voire que Fodéré à personnellement connus et il faut supposer que les «livres » désignés génériquement sont plutôt des imprimés. Ainsi, à Dole, il vante le P. Claude de La Barre, qu'il a fréquenté dans les années 1590, "père des bonnes lettres » qui a fait une «Bibliotheque des plus magnifiques en son bastiment, mais des mieux meublees et enrichie de livres de toutes sciences, lesquels outre le tres-grand nombre sont tous rares et singuliers, en quoy il n'a rien espargné pour en recouvrer de tous les païs estrangers avec une grande curiosité et despense non pareille ${ }^{23}$. Cette mémoire peut toutefois être plus ancienne encore. À propos du couvent de Romans, il rappelle le rôle joué par le frère André Edoüard au XIIIe siècle, qui «à son entrée de religion donna deux mille florins Dauphinois pour faire la Bibliotheque, ainsi qu'il se lit encore dans un escriteau en pierre qui est plombé en la muraille $»^{24}$.

Le passage de la mémoire à l'histoire est rendu possible par l'inscription de ces collections dans des rythmes historiques clairement définis, liés soit à l'histoire des frères mineurs, notamment la grande secousse des années 1510 avec le triomphe de l'observance, soit aux dégâts causés par la Réforme. Fodéré est un historien et ces aspects ne lui ont pas échappé. Il insiste ainsi sur les variations et les vicissitudes que ces collections ont connues, qui historicisent aussi ces « objets » et les inscrivent dans une temporalité qui peut à son tour faire surgir le manuscrit médiéval comme catégorie spécifique de livres. Signe de cette historicisation, peut-être consciente, l'auteur change de vocabulaire selon qu'il évoque les collections ou locaux du Moyen Âge («librairie ») ou ceux qui lui sont contemporains («bibliothèque »). Il a ainsi parfaitement intégré une mutation des usages lexicographiques qui a lieu au début du XVIIe siècle ${ }^{25}$. À ClermontFerrand, par exemple, au tournant des XVe et XVIe siècle, Fodéré estime que la «librairie » était «meublee de bons livres ${ }^{26}$, ce qui suggère qu'elle ne l'est plus. À Beaune, le gardien Jean Guilleton, à la fin du XVe siècle, "voyant que la Librairie estoit du tout destituée de Livres, il la convertit en un Réfectoir ${ }^{27}$. Le passage des couvents de Conventuels à l'observance a pu aussi faire du tort aux collections, comme il l'observe à Mâcon, où les religieux laissant place aux observants ont quitté le couvent avec les archives et « les livres tant de la Bibliotheque que de Chanterie » 28 .

Il insiste aussi sur les aléas dont sont victimes les bibliothèques. À Chalon, par exemple, la bibliothèque médiévale était superbe, elle était à la fin du XV siècle "supportee par deux voustes, en laquelle on entre depuis le milieu du dortoyr, \& laquelle estoit meublee de fort beaux pulpitres \& tableaux de chesne chargés d'un grand nombre de tous livres qui estoient en vogue en ce temps là : mais ils furent partie pillez, le reste bruslez aux premiers troubles ${ }^{29}$. Cette observation attire aussi notre attention sur la péremption dont le manuscrit peut faire l'objet: il devient, aux yeux de ses

\footnotetext{
22 Ibid., p. 736. Nous soulignons.

23 Ibid., p. 661.

24 Ibid., p. 622.

25 F. HENRYOT, op. cit., p. 197.

${ }^{26}$ J. FODÉRÉ, Narration historique..., p. 512.

27 Ibid., p. 445.

28 Ibid., p. 422.

${ }^{29}$ Ibid., p. 749. Nous soulignons.
} 
possesseurs, un objet respectable par son antiquité mais plus vraiment utile à l'apostolat quotidien. À Lyon, enfin, la bibliothèque est brûlée par les calvinistes en $1562^{30}$. Les conflits confessionnels n'ont pas toujours endommagé les bibliothèques et il arrive que les religieux chassés des terres gagnées à la Réforme puissent partir de manière ordonnée, avec leurs archives et leurs livres, ces derniers n'étant pas particulièrement visés par la fureur destructrice des calvinistes. Cette nuance montre clairement que Fodéré a su placer la bibliothèque au rang des objets historiques, qu'il examine sans passion et sans préjugés.

Cette capacité à historiciser les bibliothèques médiévales est visible dans d'autres ordres. Chez les dominicains par exemple, les chroniques conventuelles rédigées au XVII ${ }^{\text {e }}$ siècle témoignent de la mise en place d'une mémoire d'un "âge d'or » intellectuel médiéval que les destructions causées par les guerres de religion ont anéanti. Cette césure chronologique, qui est du reste bien réelle, est sensible sous la plume de Charles Gaud, prieur du couvent de Lyon entre 1676 et 1679, qui rapporte le pillage de la bibliothèque par les Huguenots en 1562 : « Les protestants [...] pillèrent la sacristie, la bibliothèque, les archives, chambres, greniers, caves et offices ; ils firent pourtant inventaire de tout par main de notaire, excepté de la bibliothèque, parce qu'ils en étaient comptables par devant l'impie et cruel baron des Adrets et led. Baron par devant le prince de Condé. Il commit un libraire nommé Guillaume Gazeau pour faire inventorier les reliquaires, argenteries et ornements [...]. Il ne conste pas ce que devinrent ny les ornements d'église, ni les livres de la bibliothèque ; mais par effet tout fut perdu pour nos religieux et par le procès instruit en grief qu'ils présentèrent contre le Sr des Adrets prisonnier à Lyon après l'Édit, ils se plaignirent d'avoir perdu [...] une des plus belles bibliothèques qui fut dans l'église de Dieu et surtout considérable par son grand nombre de divers manuscrits et originaux $»^{31}$. On devine sous sa plume la conscience d'un patrimoine perdu - rappelons que les dominicains de Lyon, grâce à leurs précieux manuscrits, avaient accompagné la publication de divers ouvrages lorsque la presse typographique avait fait son apparition à Lyon.

Jean de Réchac, également dominicain, historiographe et hagiographe de son ordre au milieu du XVIIe siècle, est encore la manifestation d'une approche historique des bibliothèques médiévales de son ordre. Se penchant par exemple sur l'histoire du couvent de Metz ${ }^{32}$, fondé en 1215, il rappelle que les bâtiments sont sortis du sol en 1221 : l'église et à sa gauche, un grand corps de logis au premier étage duquel se trouvait la Librairie, au-dessus de la cuisine et du réfectoire. Il met surtout en exergue quelques figures marquantes de l'histoire de la bibliothèque de ce couvent aux XIII e et XIve siècles. Henri de Metz, par exemple, fit venir deux volumes de commentaires de Job, d'Isaïe, des Prophètes et de Jérémie ; Jean de Trêves, un manuscrit d'Aristote. Mais c'est surtout à Bernard de Metz, dans la seconde moitié du XIIIe siècle, que la bibliothèque devait sa richesse : il avait fait venir 35 livres de théologie et de philosophie, sans compter « tous les euvres de saint Augustin en divers volumes doublez et redoublez pour l'usage des frères ». Frères qui avaient gardé une certaine reconnaissance pour leur bienfaiteur, puisque dans tous les missels de la maison, ils avaient ajouté dans la marge du second memento le nom de Bernard de Metz, pour que les générations suivantes n’oublient pas son mérite. Vers 1315, le P. Guérin « excita son Père Comte de Bar à multiplier la

\footnotetext{
30 Ibid., p. 491.

${ }^{31}$ A.D. Rhône, 6H9. Cité par J.-D. Levesque, Les frères prêcheurs de Lyon : Notre-Dame de Confort, 1218-1789, Lyon, 1978, p. 221-222.

32 J. de RÉCHAC, La Vie du glorieux patriarche S. Dominique [...]. Avec la fondation de tous les couvents et monastères de l'un et l'autre sexe, Paris, 1647, p. 878-883.
} 
Librairie de beaucoup de volumes »; d'autres encore ajoutèrent de nouveaux manuscrits à ce fonds remarquable. Ainsi, à la fin du XVe siècle, la bibliothèque était considérée comme une des plus belles de l'ordre. L'exégèse et la patristique étaient les deux disciplines les plus représentées avec respectivement quinze et dix titres; et les grands textes philosophiques du Moyen Âge, avec Averroès, Avicenne et Boèce. La théologie accordait une large place au Livre des sentences et à ses commentaires. L'hagiographie et le droit intéressaient moins les religieux, qui disposaient cependant des sommes indispensables en ces domaines: la Legenda sanctorum, les Vitae Patrum pour l'édification ainsi que le Digestum novum et les Martiniana decreti en droit canon. Le déclin qui frappa le couvent au cours du XVe siècle, et pour plusieurs décennies, dut faire quelque tort à cette collection. Le couvent avait renoué avec l'observance lorsque la ville fut assiégée par Charles Quint en 1552. Commença alors une période troublée, où les religieux abandonnèrent provisoirement leur maison et la laissèrent aux mains des bénédictins de Saint-Arnould. Jean de Réchac passe sous silence ces difficultés et leurs conséquences probables sur la bibliothèque. Il est certain, toutefois, que ces manuscrits avaient disparu de la bibliothèque au moment de la restauration des dominicains à Metz au XVII ${ }^{\text {e }}$ siècle. Replaçant ainsi la bibliothèque dans le temps, un temps scandé par les arrivages de manuscrits, Jean de Réchac se montre conscient des temporalités auxquelles ces collections sont soumises. Il en va de même à propos des dominicaines de Toul, pour lesquelles il rappelle que monsieur de Fleury, chanoine de la cathédrale et frère de deux Prêcheresses de la ville, avait offert « ses livres en françois qui etoient en bon nombre $»^{33}$ aux religieuses. Toutefois, la tentative de Jean de Réchac pèche encore par l'absence de systématisme; il laisse de côté bien des bibliothèques pourtant importantes, comme celle de Verdun.

Cette relative indifférence des religieux aux manuscrits médiévaux tient à la définition même de «bibliothèque » dans les ordres mendiants. C'est en effet au XVII siècle que s'affirme chez eux, et à contre-courant de la patrimonialisation balbutiante des collections manuscrites chez les laïcs et dans les plus prestigieux établissements réguliers, la fonction de la bibliothèque comme lieu de documentation professionnelle, et non pas comme espace favorable à l'érudition. Dans les constitutions des différents ordres mendiants, la question de la bibliothèque est traitée avec celle de l'usus rerum (la pauvreté) ou des études et de la prédication. Du reste, le lecteur en théologie et le bibliothécaire ne sont souvent qu'une seule et même personne. Cette conception de la bibliothèque n'est pas propre à mettre en évidence la place des manuscrits, nettement moins utiles aux religieux que les sermonnaires récents, les recueils de cas de conscience de la casuistique revisitée au XVII ${ }^{e}$ siècle, ou des livres spirituels qui envahissent et le marché du livre, et les bibliothèques ecclésiastiques. Le récollet Placide Gallemant témoigne bien de cette perception des collections de livres dans son ordre. Dans sa topographie de la province de Saint-Denis, il décrit 22 couvents, omettant rarement d'évoquer leur bibliothèque, mais pour deux d'entre elles seulement il fait allusion à des «trésors" patrimoniaux: au couvent de Verdun, il mentionne un pontifical de Strasbourg manuscrit, sur parchemin, avec des enluminures très soignées en marge ${ }^{34}$; au couvent de Paris, la bibliothèque est également remarquable ${ }^{35}$. Ailleurs, il se contente, avec une formule répétitive, de signaler que les livres sont nombreux, et

\footnotetext{
33 Ibid., p. 900.

34 P. GALLEMANT, Provincia sancti Dionysii fratrum minorum recollectorum in Gallia a venerando Patre Placido Gallemant eiusdem Provinciae Diffinitore, Châlons, 1649, p. 81.

35 Ibid., p. 126-127.
} 
que les prédicateurs et lecteurs en théologie et en philosophie y trouvent tout ce dont ils ont besoin ${ }^{36}$.

Chez Jean de Réchac ou Placide Gallemant, la plupart des bibliothèques échappent donc à la formulation d'une appréciation, quand même ils contiennent des manuscrits médiévaux. Cette façon de procéder, reposant sur la réputation publique ou interne à l'ordre, de quelques rares collections, est toutefois intéressante en ce qu'elle dessine une carte "mentale», imaginée et non pas réelle, des collections par les religieux, qui préjugent des bibliothèques en fonction de ce qu'on en dit ordinairement et non pas d'une visite érudite, comme le feront au siècle suivant les religieux auteurs de Voyages littéraires ${ }^{37}$. La "carte mentale» qui surgit du Traicté des plus belles bibliothèques publiques et particulières (Paris, 1644) de Louis Jacob, religieux carme déjà évoqué, est à ce titre tout à fait intéressante (document 1). Selon ses critères se dessine une répartition géographique et institutionnelle des collections conventuelles qui n'est pas réaliste mais qui est significative d'une certaine manière d'appréhender le patrimoine des couvents pour un religieux. D'abord, les bibliothèques de couvents mendiants sont aussi nombreuses à être décrites que celles des abbayes, prieurés ou chartreuses, signe d'une méconnaissance car au milieu du XVIIe siècle, les ordres monastiques et canoniaux sont déjà mieux pourvus en livres que les couvents ${ }^{38}$, surtout sur la base du seuil de 3000 volumes qu'il préconise ${ }^{39}$. Ce premier déséquilibre montre clairement la volonté de faire la publicité d'une certaine manière "mendiante» d'accumuler des livres et de les rassembler en bibliothèque organisée, qui vaut bien celle des fils de saint Benoît, de saint Bernard ou de saint Norbert.

D'autres territoires du livre se dessinent au sein des mendiants. Il cherche à valoriser les bibliothèques de son ordre, qui sont les plus nombreuses à être énumérées, à l'échelle européenne comme à l'échelle française (cf. documents 1 et 2). Il s'agit, certes, de celles qu'il connaît le mieux et selon sa méthode de travail, celles qui ont pu le plus aisément lui être données à connaître. Mais cette disproportion laisse soupçonner une volonté non clairement avouée de présenter son ordre comme un ordre intellectuel. Cette démonstration est toutefois étonnante, car les minimes comptabilisent selon les critères de Louis Jacob beaucoup de bibliothèques en France, alors qu'il s'agit d'un ordre à l'origine clairement anti-intellectuel. Jacob semble projeter sur les disciples de François de Paule les évolutions récentes et extrêmement localisées vers les études scientifiques, incarnées par le P. Mersenne par exemple ${ }^{40}$. Du reste, à ses yeux, seules les bibliothèques françaises de cet ordre sont intéressantes à ses yeux.

\footnotetext{
${ }^{36}$ Ibid., p. 69 (Metz), p. 95 (Nevers), p. 108 (Châteauvillain), p. 120 (La Charité-sur-Loire), p. 134-135 (Saint-Denis), p. 143 (Gisors), p. 152 (Châlons), p. 158 (Montereau), p. 168 (Saint-Germain), p. 173 (Rouen), p. 177 (Clamecy), p. 182 (Vitry-le-François), p. 187 (Chaumont). Les adjectifs utilisés pour caractériser les livres de ces bibliothèques sont: « commodes», « suffisants » ou « nombreux », « utiles », «nécessaires ».

37 Voir le cas le plus célèbre, celui d’U. Durand et Ed. Martène, Voyage littéraire de deux religieux bénédictins de la congrégation de Saint-Maur, Paris, 1717-1724. Ce texte a été étudié par Daniel-Odon Hurel, « La place de l'érudition dans le Voyage littéraire de dom Edmond Martène et dom Ursin Durand (1717 et 1724) », Revue Mabillon, 3 (1992), p. 213-228.

$38 \mathrm{Cl}$. JOLLY, «Unité et diversité des collections religieuses », Cl. Jolly (dir.), Histoire des bibliothèques françaises. Les bibliothèques sous l'Ancien Régime. 1530-1789, Paris, 1988, p. 11-27.

39 Selon les dossiers des archives nationales (F17 1168-1179b), en 1791, sur 1249 bibliothèques de couvents mendiants masculins énumérées, seulement 128, soit 10, 2 \%, dépassent les 3000 volumes, et la moyenne nationale est de 1431,2 volumes par couvent.

40 Sur Marin Mersenne, la bibliographie est abondante. Voir en dernier lieu A. Ruellet, « Le 'grand négociant des lettres' : contours, évolution et usages du réseau épistolaire de Mersenne », A. Vauchez et B. Pierre (dir.), Saint François de Paule et les minimes en France (fin du XVe - XVIIIe siècles), Tours, 2010, p. 279-289.
} 


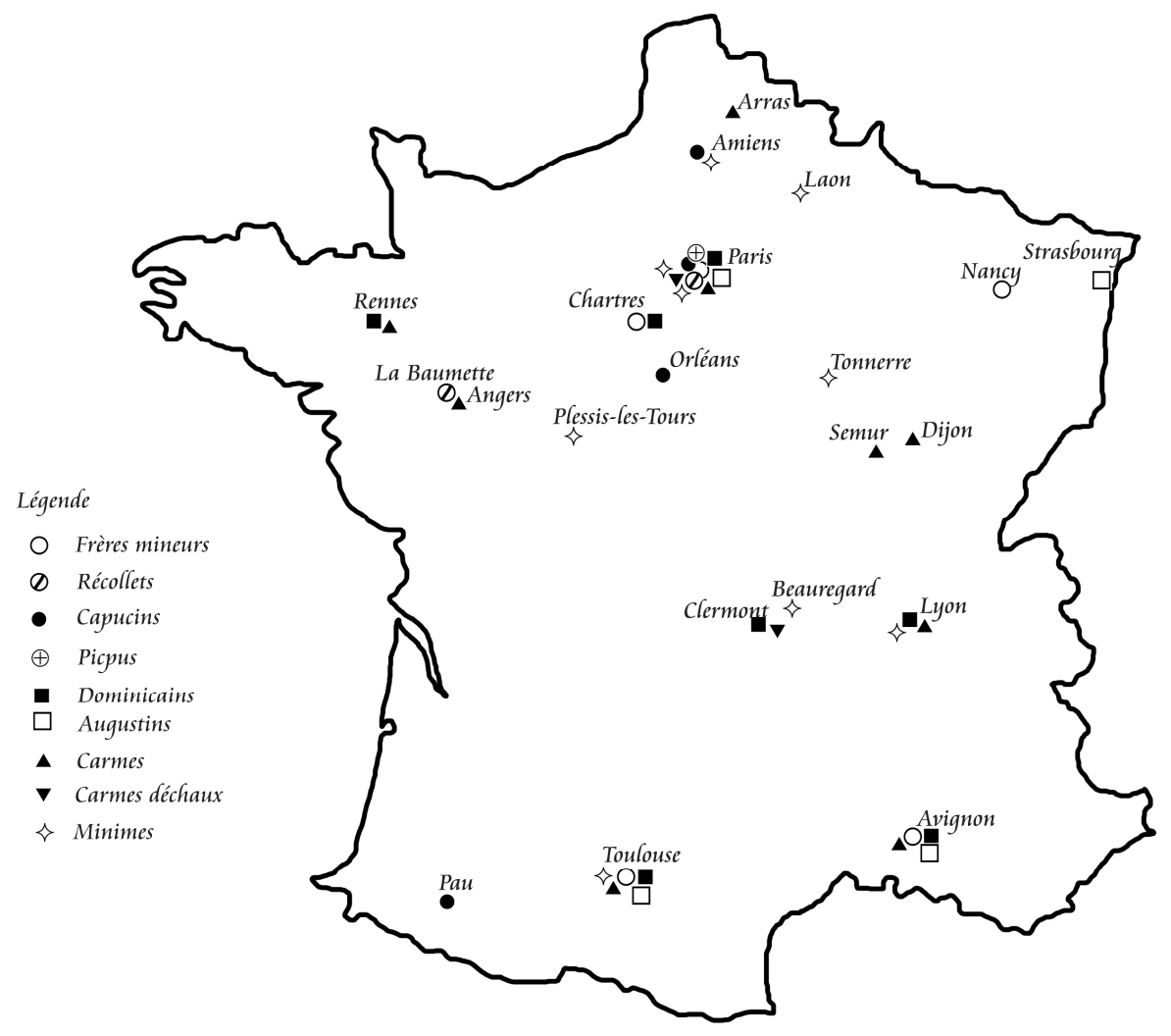

D’une manière générale, les belles bibliothèques mendiantes sont en Italie ou en Espagne, comme c'est particulièrement le cas pour les dominicains (12 bibliothèques italiennes décrites pour 7 françaises), les augustins ( 6 collections italiennes décrites pour 4 françaises) ou les frères mineurs ( 8 collections italiennes décrites pour 5 françaises).

Document 2 : répartition des bibliothèques de couvents mendiants mentionnées par Louis Jacob (1644)

\begin{tabular}{|l|c|c|}
\hline Ordre & Europe & France \\
\hline Augustins & 16 & 4 \\
\hline Capucins & 7 & 4 \\
\hline Carmes & 31 & 9 \\
\hline Carmes déchaux & 5 & 2 \\
\hline Dominicains & 27 & 7 \\
\hline Frères mineurs & 19 & 5 \\
\hline Minimes & 10 & 9 \\
\hline Récollets & 5 & 2 \\
\hline Tertiaires réguliers & 1 & 1 \\
\hline
\end{tabular}

À l'intérieur du territoire « français ${ }^{41}$ se dessinent à nouveau des vides et des pleins. L'Est est quasiment vide, et il ne cite que les bibliothèques des cordeliers de Nancy et celle des augustins de Strasbourg, laissant de côté les importantes

${ }^{41}$ Par commodité, la Lorraine, la Savoie et Avignon sont intégrées au territoire français dans ce tableau, mais elle n'en font pas partie au milieu du XVIIe siècle. 
bibliothèques champenoises par exemple, et non sans commettre des erreurs monumentales : à propos de la bibliothèque des cordeliers de Nancy, il écrit : "Dans le convent des Religieux de l'Observance de S. François de Nancy est conservée la très splendide bibliothèque des Ducs de Lorraine, depuis un long temps: la quelle est abundante en bons et rares livres, qui y ont esté mis avec de grands soins. On y void aussi quelques manuscrits $»^{42}$. En réalité, les bibliothèques des ducs et des frères mineurs étaient parfaitement distinctes mêmes si le palais et le couvent étaient voisins et cette confusion est révélatrice d'une façon d'écrire par ouï-dire. Les bibliothèques de l'ouest n'ont guère plus de faveur, à part celles des deux couvents rennais des dominicains et des carmes. Au nord, seules les villes d'Amiens, Laon et Arras peuvent s'enorgueillir de «belles» bibliothèques conventuelles. Paris s'impose en revanche comme la capitale des livres autant que des couvents. Chaque ordre y a une importante collection et il décrit dix bibliothèques. Avignon (cordeliers, dominicains, carmes, augustins), Toulouse (dominicains, carmes, minimes, augustins) et Lyon (dominicains, minimes, carmes) viennent ensuite. Dans le centre et l'ouest de la France, c'est à Chartres, Clermont, Angers et Rennes que se trouvent les plus belles collections.

En somme, cette géographie est totalement fantaisiste. Elle montre en revanche la sensibilité de Louis Jacob à l'histoire de chaque ordre, pointant avec précision les couvents primitifs, comme celui de Plessis-Lès-Tours pour les minimes, où était mort François de Paule. La bibliothèque des carmes de Rennes, quant à elle, est associée à la réforme de l'ordre, née dans ce couvent au début du XVIIe siècle.

Le vocabulaire employé par Louis Jacob est tout aussi révélateur d'une hiérarchisation des collections. Qu'elles soient "notables », "curieuses », "réputées », " considérables», "insignes» ou "recommandables», les manuscrits contribuent particulièrement à leur valeur culturelle. Chez les carmes de Semur-en-Auxois, par exemple, «CXX manuscrits » voisinent avec plus de 4000 volumes imprimés ${ }^{43}$. Dans les couvents toulousains, "outre les livres imprimez, il y a plusieurs anciens mss. dans icelles ${ }^{44}$. Et ainsi de suite, chez les dominicains de Chartres, dont la bibliothèque " est de grande considération pour le nombre de ses livres, entre lesquels il y a beaucoup de manuscrits $»^{45}$.

La conjonction la plus intéressante selon Louis Jacob, entre bibliothèques conventuelles et manuscrits, tient à la pérennité des premières dans la conservation de collections réunies par de «sçavans personnages, afin que la mémoire ne s'en perde ${ }^{46}$. Il évoque alors la bibliothèque des minimes de Tonnerre, qui a bénéficié de la générosité de Charles-Henri de Clermont-Tonnerre, qui a donné aux frères tous les manuscrits de sa famille ${ }^{47}$. Le grand mérite de la bibliothèque des dominicains d'Avignon est de conserver les manuscrits de la collection rassemblée par le pape Jean XXII. C'est aussi le cas - entre les lignes - de la très réputée bibliothèque du couvent récollet de La Baumette près d'Angers, dont le carme écrit qu'elle est "assés bonne \& curieuse " ${ }^{48}$, sans préciser qu'il la mentionne à cause des manuscrits que le roi René d'Anjou a offerts

\footnotetext{
42 L. JACOB, Traicté sur les plus belles bibliothèques..., t. II, p. 436.

43 Ibid., p. 635.

44 Ibid., p. 654.

45 Ibid., p. 689.

46 Ibid., « au lecteur» (non paginé).

${ }^{47}$ Ibid., p. 636. Sur ce don, voir P. GASNAULT, « Charles-Henri de Clermont-Tonnerre et la bibliothèque du couvent des minimes de Tonnerre », Du copiste au collectionneur. Mélanges d'histoire des textes et des bibliothèques en l'honneur d'André Vernet, Turnhout, 1998, p. 585-614.

48 L. JACOB, op. cit., p. 606.
} 
au couvent. Avec Louis Jacob, la notion de transmission est inhérente à celle de bibliothèque conventuelle, grâce à son caractère transgénérationnel et propre à défier le temps.

Cette carte subjective nous renseigne donc davantage sur les a priori de Louis Jacob en matière de bibliothèques, que sur les bibliothèques elles-mêmes. S'il est impossible de dresser une carte plus objective, la projection topographique des données recueillies dans les relevés de sources ${ }^{49}$ ou les inventaires contemporains de manuscrits ${ }^{50}$ permet de préciser ce tableau, malgré d'évidentes lacunes (ne sont mentionnés que les sources ou manuscrits ayant survécu) et les difficultés d'interprétation (il n'est pas toujours possible de déterminer à quelle date un manuscrit est entré dans la bibliothèque). Sur la base d'une démarche similaire, Marie-Louise Auger a pu poser l'hypothèse de l'existence de 37 bibliothèques franciscaines au moins à la fin du Moyen Âge (c'est-à-dire des fonds qui comportaient d'autres livres que les traditionnels bréviaires, bibles, règles et commentaires de celle-ci), notamment à Lonsle-Saunier, Salins, Neufchâteau, Verdun et Châlons-sur-Marne ${ }^{51}$. Malgré leur caractère fragmentaire, ces données révèlent donc, même grossièrement, quelques gros ensembles documentaires médiévaux ayant connu une certaine stabilité dans les bibliothèques conventuelles à l'époque moderne.

C'est le cas des cordeliers de Dole dont le couvent fut fondé en 1372, couvent jamais cité par les historiens anciens et les topographies, mais pour lesquels le Catalogue général des manuscrits recense 34 manuscrits médiévaux ${ }^{52}$. Ces manuscrits sont par ailleurs connus par une liste de livres prêtés aux religieux des Thons en 148953 et semblent être revenus, puis avoir été conservés de manière satisfaisante dans ce couvent jusqu'à la fin du XVIIIe siècle. C'est encore le cas pour les cordeliers de Troyes, dont la bibliothèque est assez bien documentée par des inventaires établis entre 1528 et 1769 et qui conservait un nombre important de manuscrits, depuis le Moyen Âge et jusqu'à la Révolution. Cette bibliothèque n'est jamais citée par les topographes, religieux ou laïcs alors qu'elle a été une des premières bibliothèques publiques de France à partir de 1651 et qu'une dizaine de manuscrits anciens au moins sont parvenus jusqu'à nous ${ }^{54}$. Enfin, une interrogation du Catalogue général des manuscrits met en évidence la bibliothèque des carmes de Semur-en-Auxois, cette fois parfaitement connue de Louis Jacob, pour lesquels subsistent au moins 24 manuscrits anciens ${ }^{55}$, certains d'entre eux n'étant toutefois arrivés au couvent qu'au cours du XVIe siècle, par le truchement d'un certain frère Étienne Bridolet ${ }^{56}$.

Louis Jacob a également omis des bibliothèques de villes importantes, comme celle des dominicains de Dijon, celle des dominicains de Langres, ou des collections aussi prestigieuses de celle des dominicains de Saint-Maximin en Provence, que les religieux

\footnotetext{
49 A.-M. GENEVOIS, J.-F. GENEST et A. CHALANDON, Bibliothèques de manuscrits médiévaux en France : relevé des inventaires du VIIIe au XVIIIe siècle, Paris, 1987.

${ }^{50}$ Catalogue général des manuscrits des bibliothèques publiques de France [en ligne], désormais CGM.

51 M.-L. AUGER, «La bibliothèque des Cordeliers de Troyes », Hommage à J. Vieillard, bulletin de l'Institut de Recherche et d'histoire des textes, 15 (1969), p. 183-250.

52 CGM, t. 21, B.M. Lons-le-Saunier, , ms. 1 ; CGM, t. 13, Dole, 33 ms.

53 G. DUHEM, «Quelques documents du fonds des cordeliers de Dole », Mémoires de la Société d'Émulation du Jura, 10 (1941), p. 40-42.

54 M.-L. AUGER, op. cit., ; voir aussi J.-G. Bougerol, Les manuscrits franciscains de la bibliothèque de Troyes, Grottaferrata, 1982.

55 CGM, t. 6, Semur-en-Auxois.

56 B.M. Semur-en-Auxois, ms. 15 (15) : Recueil de traités de théologie et d'homélies, XIIe-XIIIe siècle ; ms. 21 (21) : Incipit abrevatio operis carmelite..., XVe s. ; ms. 26 (27) : Prologus Paterii in libris veteris et novi testamenti, XII ${ }^{\mathrm{e}}$ siècle.
} 
tenaient du roi René57. Certes, ces bibliothèques n'atteignaient peut-être pas les 3000 volumes requis pour figurer dans le Traité, mais leur réputation n'était plus à faire au XVII siècle.

En ce siècle donc, le manuscrit possède aux yeux des mendiants un statut ambigu. Il ne fascine pas beaucoup, alors même que d'autres milieux culturels, laïcs ou princiers, cherchent à en acquérir pour rehausser leurs bibliothèques. Pour les religieux, le manuscrit est finalement un livre comme un autre, faute peut-être de posséder les clefs nécessaires à la compréhension de ces documents (paléographie, mise en texte), hormis dans les cas, assez rares, de religieux avertis. Il est alors paradoxal de constater qu'en dépit de cette indifférence, les couvents de fondation post-tridentine, notamment capucins et minimes, ont renfermé dans leurs toutes nouvelles bibliothèques des manuscrits médiévaux.

\section{La circulation des manuscrits}

Le Catalogue général des manuscrits met aussi en évidence un paradoxe : la constitution par les ordres nouveaux, de fondation postérieure à 1580, de fonds de manuscrits médiévaux dont l'usage pastoral ou théologique est en décalage avec les prescriptions faites en ce domaine dans leurs constitutions. Chez les minimes de Beaune, par exemple, installés dans la ville en 1627, se trouvaient au moins trois manuscrits antérieurs au XVe siècle : une compilation d'extraits de Lactance et d'Ovide du XVe siècle

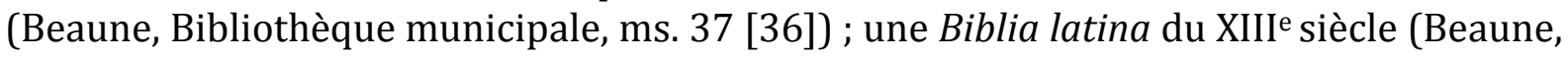
Bibliothèque municipale, ms. 57 [56]) et une Summa de penitentia fratris Antonini de Florentia de la fin du XV ${ }^{\text {e }}$ siècle (Beaune, Bibliothèque municipale, ms. 200 [111]). Ce dernier ouvrage est arrivé dans ce couvent en 1675 si on en croit une mention manuscrite portée dans l'ouvrage. L'existence de documents très anciens dans les bibliothèques modernes est également attestée par les premiers historiens de ces ordres nouveaux, tel Jean-Marie de Vernon, qui dans son ouvrage sur l'histoire du Tiers-ordre régulier de saint François, évoque le couvent de Nancy, où « la bibliotheque est pleine de beaux et de bons livres, voire de manuscrits, particulièrement une histoire de Lorraine non encore imprimée, d'un frère Richier moine qui estoit du temps de sainte Elizabeth dont il parle ${ }^{58}$. Il fait allusion à la chronique de Richer, religieux bénédictin de Senones (vers 1190-1266), qui a tenu la chronique des événements de son époque ${ }^{59}$. Le cas de Nancy témoigne d'ailleurs parfaitement de ce que ce ne sont pas toujours les couvents fondés au Moyen Âge qui sont les mieux pourvus en documents anciens, puisque les cordeliers n'en ont pas mais que les capucins ou les tertiaires réguliers en possèdent (document 3).

\footnotetext{
57 B. MONTAGNES, «La Bibliothèque de St-Maximin en 1508 », Livres et bibliothèques (XIIIe-XVe siècle), Toulouse, 1996 (Cahiers de Fanjeaux 31), p. 241-259.

58 Jean-Marie de Vernon, Histoire générale et particulière du Tiers Ordre de saint François d'Assize, Paris, 1667, t. III, p. 212.

${ }^{59}$ Sur ce document et son histoire, voir D. DANTAND, Chronique de Richer, moine de Senones. Présentation, édition et traduction, thèse de doctorat, Université Nancy 2, 1996.
} 
Document 3 : les manuscrits dans les bibliothèques conventuelles de Nancy au XVIIIe siècle d'après les provenances signalées au catalogue des manuscrits de la B.M. Nancy

\begin{tabular}{|l|l|l|l|}
\hline Couvent & $\begin{array}{l}\text { Date de } \\
\text { fondation }\end{array}$ & Nombre de manuscrits & $\begin{array}{l}\text { Nombre de manuscrits } \\
\text { antérieurs au XVe s. }\end{array}$ \\
\hline Cordeliers & 1482 & 2 & 0 \\
\hline Minimes & 1592 & 3 & 0 \\
\hline Capucins & 1593 & 3 & 2 \\
\hline Carmes déchaux & 1611 & 6 & 0 \\
\hline Augustins & 1621 & 1 & 0 \\
\hline Tiercelins & 1622 & 26 & 4 \\
\hline Dominicains & 1641 & 0 & 0 \\
\hline
\end{tabular}

Il faut donc comprendre les modalités de circulation de ces manuscrits et de constitution de ces fonds. Dans la plupart des cas, ces enrichissements ne semblent pas intentionnels, mais sont une conséquence de la générosité des laïcs à l'égard des religieux mendiants, générosité qui concerne aussi bien le bâtiment, l'alimentation, le vêtement, les objets du culte que les livres. Cette générosité est ancienne et existait déjà au temps où le manuscrit était l'unique mode de reproduction et de diffusion de l'écrit. À Nancy, par exemple, le duc René II avait fait beaucoup pour " ses » cordeliers qu'il avait installés dans sa capitale en 1482 ; dans leur nécrologe rédigé en 1764, les religieux n'oublièrent pas de rappeler la mémoire de ce prince qui «avait ordonné que les appartements à l'usage des frères et leurs cellules fussent décemment disposés, la bibliothèque enrichie de livres de toutes sortes » 60 , en particulier une « bible in-quarto, de vélin, reliée de maroquin rouge sur tranche rouge, escrite en gothique et enrichie de lettres et vignottes en mignature ». La première page de cette Bible était signée de la main du duc. Mais à l'époque moderne, ces fondateurs et bienfaiteurs, eux-mêmes collectionneurs, continuèrent d'offrir leurs manuscrits aux religieux mendiants, alors même que ceux-ci n'en avaient plus vraiment l'usage, surtout quand ils pouvaient compter sur des éditions imprimées plus commodes à consulter. Au couvent des capucins de Bourg-en-Bresse, qu'il avait fondé en 1612, le sieur de Rogemont donne ainsi un Livre du sainct Graal du XIII ${ }^{\mathrm{e}}$ siècle ${ }^{61}$, qui n'est pas précisément nécessaire aux religieux mais dont la conservation perpétuelle est désormais garantie pour l'ancien possesseur. Il est possible que les deux autres manuscrits anciens décrits pour ce couvent aient la même provenance ${ }^{62}$. Aux yeux de ce donateur, comme aux yeux de tous les contemporains, le livre, manuscrit ou imprimé, est un objet culturel générique. La plupart du temps, en effet, ils lèguent aussi bien l'un que l'autre type d'ouvrage, sans préférence. Le même Rogemont fit don de livres imprimés, notamment une Histoire d'Italie de Messire François Guicciardin gentilhomme florentin (Paris, 1567) ${ }^{63}$.

Les exemples sont infinis. Henriette de La Guiche, épouse de Philippe de Valois duc d'Angoulême, a légué au couvent des minimes de La Guiche en Bourgogne, qu'elle avait fondé, une riche collection de manuscrits venus de la famille de son mari. On y trouve, entre autres, une Cité de Dieu du XVe siècle, une Légende dorée, un récit de la

\footnotetext{
60 Abbé GUILLAUME, « Le couvent des cordeliers et la chapelle ronde de Nancy », Bulletin de la Société d'Archéologie Lorraine, 1850, p. 74.

${ }^{61}$ CGM, t. 6, B.M. Bourg-en-Bresse, ms. 55(45) : Ci commence li livres du sainct Graal qui à proprement parler est dit Joseph d'Aharimathie..., XIII" siècle.

62 Ibid., ms. 6(4) : [Pierre Lombard, commentaire sur les Psaumes], XIIIe siècle; ms. 8(6) : Bernard de Pavie, Commentaire sur les décrétales, 1342.

63 Exemplaire B.M. Lyon, 24774.
} 
Toison d'or de la fin du XVe siècle ${ }^{64}$. Les capucins de Salins, fondés en 1582 par le chanoine Pierre Moreau, du chapitre Saint-Jean-Baptiste de la même ville, bénéficièrent d'un don important de leur fondateur, bibliophile éminent, de manuscrits, incunables et imprimés précieux à la fin du XVI ${ }^{\mathrm{e}}$ siècle, avant que le maire de la ville, François Merceret, ne charge les religieux de tenir une bibliothèque publique en 159365 . De la sorte, les religieux se sont vus remettre au moins onze manuscrits, actuellement conservés à la Bibliothèque de la ville ${ }^{66}$. Entre couvents aussi, le livre circule. À Besançon, les clarisses se dessaisissent de quelques manuscrits précieux pour les remettre aux capucins de la ville, dont le couvent a été fondé en 1607, notamment une Somme des cas de conscience de Barthélemy de Pise et un traité sur la condition humaine d'Innocent III ${ }^{67}$. Ces capucins assurent en effet chez les clarisses le service liturgique et spirituel, fournissant aux religieuses des confesseurs et des directeurs de conscience, et participant à l'entretien de la mémoire de Colette de Corbie, réformatrice des clarisses de la ville en 1410. Ces liens spirituels se doublent donc d'échanges matériels. À une date inconnue, postérieure à 1628, les minimes de Metz inscrivent leur ex-libris sur une copie des Évangiles selon le texte de la Vulgate, manuscrit du XVe siècle qui avait préalablement appartenu aux cisterciennes de Vergaville ${ }^{68}$.

Finalement, sous l'Ancien Régime, les bibliothèques des couvents mendiants offrent un tableau contrasté du point de vue des manuscrits. Les religieux, dans l'ensemble, ont revendiqué jusque dans leur rapport au livre la sobriété, sinon la pauvreté absolue, interdisant de se livrer au commerce et à l'acquisition de manuscrits précieux, mais la perméabilité de la frontière entre l'univers conventuel et celui des laïcs a permis aux manuscrits anciens d'entrer dans les collections de couvents de fondation post-tridentine.

\section{La gestion des fonds}

Ces auteurs parlent tous de «livres» indistinctement, qu'il s'agisse de manuscrits ou d'imprimés. Cette indifférenciation suggère une incompréhension de la spécificité du manuscrit et des modes de conservation particuliers dont il peut faire l'objet. On peut compter sur près d'une vingtaine de catalogues de bibliothèques conventuelles dressés au cours du XVII ${ }^{\mathrm{e}}$ siècle en France. La pratique catalographique a bien sûr été plus importante mais ces documents ont rarement survécu, puisque les inventaires de bibliothèques étaient parfois détruits lorsque de nouveaux étaient dressés. Cette source permet de déterminer le traitement bibliothéconomique et catalographique des manuscrits dans ces fonds ${ }^{69}$.

\footnotetext{
${ }^{64}$ CGM, t. 6, B.M. Mâcon, ms. 1-2: La cité de Dieu, trad. de Raoul de Presle, début du XVe siècle ; ms. 3: La Légende dorée, t. III, milieu du XVe siècle ; ms. 4-5: La Toison d'or, de Guillaume de Tournai, fin XVe siècle.

65 Voir A. POLAIN, « Note sur un livre portant un hommage d'Érasme », Mélanges offerts à Emile Picot, Paris, 1913, t. 2, p. $135-141$.

${ }^{66}$ CGM, t. 9.

67 B.M. Besançon, ms. 228: Bartholomaei de Pisis summa de casibus conscientiae, 1457 ; ms. 208 : Innocentii liber de miseria humanae conditionis, début du XIVe siècle.

${ }^{68}$ B.M. Metz, ms. 563, Quatuor evangelistae cum praefationibus Hieronymi.

${ }^{69}$ Pour une liste exhaustive de ces catalogues, voir l'annexe à notre article « Classement des livres et représentation des savoirs dans les couvents mendiants (XVIIe- XVIII siècles) », Revue française d'histoire du livre, 133 (2012), p. 8185.
} 
Document 4 : le traitement des manuscrits dans les catalogues de bibliothèques conventuelles (vers 1640 - vers 1740)

\begin{tabular}{|l|l|l|l|}
\hline Couvent & $\begin{array}{l}\text { Date } \\
\text { rédaction }\end{array}$ & $\begin{array}{l}\text { Présence de } \\
\text { manuscrits }\end{array}$ & $\begin{array}{l}\text { Présence d'une } \\
\text { section dédiée aux } \\
\text { manuscrits }\end{array}$ \\
\hline Dominicains de Toulouse & 1683 & OUI (65) & OUI \\
\hline Cordeliers de Besançon & 1667 & OUI (17) & NON \\
\hline Récollets d'Orléans & 1644 & OUI (10) & OUI \\
\hline Récollets de Corbeil & 1699 & NON & NON \\
\hline Récollets de Lyon & S.d. & OUI (13) & NON \\
\hline Capucins de Dijon & 1699 & NON & NON \\
\hline Capucins d'Orléans & 1715 & OUI (18) & OUI \\
\hline Capucins d'Angoulême & 1716 & OUI (4) & NON \\
\hline Tertiaires réguliers de Nancy & 1665 & OUI (47) & OUI \\
\hline Carmes de Bayonne & 1668 & OUI (11) & OUI \\
\hline Carmes de Rennes & 1696 & OUI (3) & NON \\
\hline Carmes déchaux de Clermont & 1669 & NON & NON \\
\hline Carmes déchaux de Chambéry & 1670 & NON & NON \\
\hline Carmes déchaux de Montpellier & 1676 & NON & NON \\
\hline Carmes déchaux de Nancy & 1696 & OUI (4) & NON \\
\hline Carmes déchaux de Lyon & S.d. & OUI (1) & NON \\
\hline Minimes de Bonsecours & 1682 & NON & NON \\
\hline Minimes d'Avignon & 1690 & NON & NON \\
\hline Minimes de La Guiche & 1740 & OUI (18) & OUI \\
\hline Minimes de Reims & Déb. XVIII siècle & OUI (3) & NON \\
\hline Minimes de Saint-Mihiel & 1723 & NON & NON \\
\hline Minimes de Lunéville & 1740 & NON & NON \\
\hline
\end{tabular}

Dans les couvents dépourvus de manuscrits ( 9 cas), le bibliothécaire n'avait évidemment pas besoin de prévoir une section pour ce type de documents. Les inventaires des carmes déchaux, par exemple ${ }^{70}$, n'en possèdent jamais mais dans la majorité des bibliothèques, il ne se trouvait pas de manuscrits anciens ou modernes, comme à Chambéry ou à Montpellier. Dans les treize autres cas, les religieux chargés du catalogage ont aussi bien mêlé les manuscrits aux imprimés ( 7 cas) que séparé ces deux types d'objets (6 cas). Malgré la petitesse de ce corpus, l'hésitation bien perceptible à l'égard des manuscrits montre que l'usage de dissocier topographiquement manuscrits et imprimés, avéré dans les documents normatifs à l'intention des réguliers dans la seconde moitié du XVII ${ }^{e}$ siècle ${ }^{71}$, a mis quelque temps à s'imposer dans la pratique. En conséquence, et comme les bibliothécaires se sont rarement donné la peine de dater les documents, nous ne pouvons savoir s'ils sont antérieurs au XVI ${ }^{\mathrm{e}}$ siècle. Ainsi, chez les carmes de Bayonne, l'inventaire précise seulement : «Libri sunt undecim manuscripti 6 philosophiae, 3 theologiae. Rhetoricae 1, devo. 1 et 1 his. Relig. »72. Chez les récollets d'Orléans, la section $\mathrm{R}$ du catalogue énumère dix volumes dont les quatre premiers,

\footnotetext{
70 G. SINICROPI, D'oraison et d'action. Les carmes déchaux en France aux XVIIe et XVIII siècle, thèse de doctorat, Université Clermont-Ferrand 2, 2010, t. 1, p. 262.

${ }^{71}$ Règles communes et particulières pour la congrégation de Saint-Maur, s. l., p. 84-87; ce chapitre sur les bibliothèques est repris mot pour mot dans le Directoire uniforme ou journal commun des officiers inférieurs de chaque convent des religieux cordeliers réformés des quatre grandes provinces de France, Paris, 1668, p. 65-67.

72 A.D. Pyrénées-Atlantiques, H 39 : Biblioteca Patrum Carmelitarum conventus Baiena Recognitaa (1e) die mensis oct. Anni 1668, non folioté.
} 
offerts au couvent par Louis Dijon, chanoine d'Orléans et écolâtre, pourraient être médiévaux mais les six autres sont, à coup sûr, modernes ${ }^{73}$.

Lorsqu'il n'existe pas de rubrique spécifique aux manuscrits dans les catalogues, il n'y avait probablement pas non plus d'armoire réservée à ce type de livres dans la pièce qui tenait lieu de bibliothèque. Pour ces collections, le bibliothécaire a mentionné des manuscrits anciens, sans s'attarder avec précision sur leur description, et les a catalogués dans les rubriques thématiques dont ils relèvent, au milieu des imprimés, sans tenir compte de leur spécificité matérielle et formelle. Chez les récollets de Lyon $^{74}$, par exemple, des manuscrits sont effectivement inventoriés dans les différentes sections bibliographiques du catalogue, simplement signalés à l'attention de l'usager par un soulignement. Le frère carme déchaussé de Lyon qui dresse le catalogue de la bibliothèque de son couvent ${ }^{75}$ signale dans la rubrique des Libri sacri un "Livre de la passion de Jésus Christ » qui pourrait être "Sy commence l'istoire de la Passion Nostre Seigneur Jhesu Crist, le benoit filz de Dieu..., laquelle à l'aide de Dieu et de la glorieuse Vierge Marie je pense ainsi poursuir et assuir », manuscrit du XVe siècle que signale le Catalogue général des manuscrits comme confisqué dans ce couvent à la Révolution ${ }^{76}$. En effet, aucun autre livre du catalogue ne porte de titre qui s'approche un tant soit peu de celui de ce manuscrit. Si tel était le cas, cet emplacement bibliographique serait intéressant car cette rubrique des Libri sacri comporte, outre les bibles, divers ouvrages que les religieux semblent désigner comme fondamentaux: les décrets du concile de Trente, quelques paraphrases bibliques alors qu'il existe une rubrique d'Expositores, des catéchismes... Il s'agit en quelque sorte du cœur normatif et dogmatique de la bibliothèque. Mais ce «Livre de la passion » n'est pas décrit comme un manuscrit.

C'est encore la pratique, au début du XVIII' siècle, du bibliothécaire en charge chez les minimes de Reims, qui n'isole pas les quelques manuscrits que possède son couvent ${ }^{77}$. Le religieux anonyme qui a rédigé le catalogue des cordeliers de Besançon en 1667 procède également de cette manière ${ }^{78}$. Quoique sa description des livres soit extrêmement sommaire, se limitant au titre et à l'auteur sans donnée d'édition ou description formelle de l'ouvrage, il a ajouté la mention «manuscriptus » à certains titres, sans qu'on puisse malheureusement savoir s'il s'agit de manuscrits modernes ou médiévaux. La dénomination de ces documents laisse à penser qu'il s'agit simplement de manuscrits scolaires (le couvent possédait depuis le Moyen Âge un studium) à l'usage des profès : «Logica manuscripta », "Cursus phylosophycus manuscriptus », ou de notes de prédicateurs. Du reste, ces 17 manuscrits ne représentent que 1,8\% des titres énumérés, proportion qui révèle une place marginale dans cette bibliothèque. Ce couvent, fondé en 1224, dispose alors d'un patrimoine écrit ancien dont il reste

\footnotetext{
73 B.M. Orléans, ms. 351(300) : Catalogus librorum bibliothecae F. M. Recollectorum Conven. Aureliae, factus anno 1644, fol. 189.

${ }^{74}$ A.D. Rhône, 7H 34 : Catalogue des livres de la bibliothèque des récollets de Lyon, s.d. L'archiviste qui a rédigé l'inventaire de la série « $\mathrm{H}$ » le situe au XVIIe siècle, datation qui nous paraît un peu précoce au vu de l'écriture et des procédés bibliographiques mis en œuvre. Nous le supposons plutôt du XVIIIe siècle.

75 B.M. Lyon, ms. Coste 1079 : Catalogus librorum conventus Lugdunensis fratrum Carmelitarum discalceatorum.

76 B.M. Lyon, ms. 864.

77 Ph. J. WHITMORE, The Order of Minims in seventeenth Century France, La Haye, 1967, p. 126-128. Parmi les manuscrits survivants, voir B.M. Reims, ms. 519 : Compendium theologicae veritatis, 1275.

${ }^{78}$ B.M. Besançon, ms. 779 : Catalogus omnium librorum bibliothecae conventus fratrum minorum conventualium sancti Francisci civitatis liberae Bisuntinae, secundum ordinum alphabeticum, numerum et materias compositus, 25 juin 1667.
} 
aujourd'hui des traces ${ }^{79}$ et il n'est pas exclu que ces manuscrits énumérés soient médiévaux.

Document 5 : les mentions de manuscrits dans la bibliothèque des cordeliers de Besançon (1667)

\begin{tabular}{|l|l|l|l|}
\hline Lettre & Intitulé de la rubrique & Nombre de volumes & Nombre de manuscrits \\
\hline A & Scripturae sacrae interpretes & 94 & - \\
\hline B & Biblia sacra & 18 & - \\
\hline C & Sancti Patres & 25 & - \\
\hline D & Theologi scolastici & 99 & 1 \\
\hline E & Jus canonicum & 30 & - \\
\hline F & Jus civile & 29 & 2 \\
\hline G & Theologi morales & 40 & - \\
\hline H & Historici & 101 & - \\
\hline I & Humanistae & 70 & - \\
\hline K & Philosophi & 64 & 8 \\
\hline L & Sermones adventus & 22 & 1 \\
\hline M & Sermones quadragesimales & 71 & 3 \\
\hline N & Sermones dominicales & 46 & - \\
\hline O & Sermones de sanctis & 61 & 1 \\
\hline P & Sermones in varias materias & 18 & - \\
\hline Q & Libri devotionis & 86 & - \\
\hline R & Libri diversarum materiarum & 63 & 1 \\
\hline
\end{tabular}

D’autres bibliothécaires, au XVIIe siècle ou au début du siècle suivant, ont isolé les manuscrits des livres imprimés, signe qu'ils ont perçu la différence matérielle et intellectuelle entre ces ouvrages. Le cas des tiercelins de Nancy est intéressant car il s'agit d'un de ces couvents modernes, chef de custodie d'un ordre restauré au début du XVII e siècle par Vincent Mussart, fondé en 1622 et qui s'impose dans la seconde moitié du siècle comme un centre intellectuel lorrain. À une date inconnue, vraisemblablement en 1665 lorsque le couvent est déplacé du faubourg de Préville à la Ville-Neuve, le P. Donat de Nancy, gardien et bibliothécaire, dresse le catalogue de la bibliothèque de son couvent, bibliothèque qu'il a grandement contribué à augmenter ${ }^{80}$. L'inventaire dénombre 2298 titres pour 2669 volumes et montre une longue et quotidienne pratique des livres ${ }^{81}$. Il est clairement conçu en fonction des usages possibles des livres, comme l'indique la répartition des rubriques catalographiques, certaines étant inhabituelles et toutefois conformes à ce qu'on sait des centres d'intérêt de ce religieux: il existe, par exemple, une section « livres d'estat et politiques » qui fait écho aux préoccupations de ce même P. Donat, religieux confesseur et conseiller du duc de Lorraine Charles IV.

Or, ce catalogue comprend une rubrique de «Manuscripti», située en fin de catalogue. Cette pratique suggère une certaine fréquentation de ce type d'objet même si le $\mathrm{P}$. Donat s'avère assez malhabile à les décrire convenablement, à la différence des imprimés. Cette rubrique rassemble 47 documents, 25 en français, 17 en latin et 4 en italien. Mais l'examen des titres laisse perplexe, car certains de ces documents ne sont sans doute pas des manuscrits, et on ne voit toutefois pas comment le P. Donat aurait pu

\footnotetext{
${ }^{79}$ CGM, t. XXXII : B.M. Besançon, ms. 4 : Biblia sacra cum S. Hieronymi prologis, milieu du XIIIe siècle ; ms. 190 : Hugues de Saint-Victor, De sacramentis liber, XII ${ }^{\text {e siècle. }}$

${ }^{80}$ Sur le P. Donat, voir F. HENRYOT, Livres et lecteurs..., op. cit., p. 201-203. B.M. Nancy, ms. 1068(657) : Inventorium generale bibliothecae fratrum tertiariorum sancti Francisci conventus Nanceiani, [vers 1665].

${ }^{81}$ F. HENRYOT, « La bibliothèque des tiercelins de Nancy au XVIIe siècle », Le Pays lorrain, 2008, n 4, p. $297-300$.
} 
les confondre avec des imprimés. L'identification de chacun de ces titres et la comparaison de ce catalogue avec celui des manuscrits de la Bibliothèque municipale de Nancy permet de formuler diverses hypothèses quant à la nature de ces documents et les raisons de leur rangement dans la section des manuscrits.

On peut d'abord suggérer que le terme «manuscrit» désigne plus généralement des documents qui ont une valeur symbolique et affective plus importante, dans ce couvent qui s'impose au XVII siècle comme un lieu de défense du patriotisme lorrain et d'attachement à la dynastie régnante en contexte d'occupation par les troupes françaises. Les imprimés rangés dans cette section sont en effet tous des imprimés lorrains, d'auteurs lorrains, de même qu'un certain nombre de manuscrits écrits par des compatriotes comme François Perrin de Dommartin, poète et héraldiste. C'est ainsi que la Relation du voyage au Levant d'Henry de Beauvau, ouvrage paru pour la première fois à Toul en 1608, se retrouve dans le rayonnage des manuscrits, aux côtés des Tablettes ou quatrains de la vie et de la mort de Pierre Matthieu également imprimées en Lorraine (Pont-à-Mousson, 1621), ou encore de L'ordre sacré de la Sainte Prestrise de Montluisant (Metz, 1633). D'autre part, ces écrivains sont tous de la génération du P. Donat, tel André du Saussay, évêque de Toul (1589-1675) ${ }^{82}$ ou de la génération précédente, comme Henry de Beauvau. Ces documents ont pu être des dons des auteurs au P. Donat, farcis de notes manuscrites ou d'hommages de la main des auteurs, et à ce titre considérés comme des objets mixtes et à forte valeur affective.

Les manuscrits les plus anciens, encore aujourd'hui présents dans les collections de la Bibliothèque municipale de Nancy, sont bien décrits dans ce catalogue, mais de manière extrêmement sommaire, sans datation, sans observations sur l'iconographie et les peintures quand il y en a. Par exemple, le De imitatione Christi libri duo du XIVe siècle ${ }^{83}$ n'est pas décrit sous ce titre, comme si le bibliographe n'avait pas identifié qu'il s'agit du traité de Thomas a Kempis, mais sous le nom Liber intimae consolationis. Incipit, dulcis Jesu memoria. En réalité, l'incipit est Dulcis Ihesu gaudia ! Le P. Donat suit donc une méthode complètement empirique pour décrire ces manuscrits, sollicitant sa mémoire plus souvent qu'il n'examine précisément les documents euxmêmes.

Il se trouve enfin un troisième type d'ouvrages dans cette rubrique, qui dépasse notre sujet mais qui permet de le mettre en perspective: d'innombrables manuscrits modernes. Parmi ceux-ci se trouvent des textes savants d'histoire ou de science, généralement des copies de documents ou traités anciens ou modernes destinés à nourrir l'érudition de tel ou tel frère. D'autre part, on remarque des notes de lecture de religieux commentant la Bible en vue de la prédication ou prenant dévotement des notes sur leur livre de piété, ou encore méditant la règle de saint François, enfin des notes de cours de lectores et d'étudiants en théologie scolastique ou morale. La copie comme exercice de dévotion est avérée également chez les tertiaires réguliers de Nancy comme dans bien d'autres couvents. Dans ce couvent lorrain, les Leçons spirituelles in quarto, des "escrits de philosophie, théologie et réthorique de divers religieux » ou encore deux volumes de Méditations in quarto appartiennent à ce genre d'ouvrages personnels et néanmoins conservés pour l'usage de tous dans la bibliothèque commune. Le manuscrit, au XVII ${ }^{e}$ siècle, est un document résolument contemporain. Né de l'imprimé, dont il reproduit parfois même la forme matérielle et imite la typographie, il n'a pas de raison de s'en distinguer dans les rayonnages des bibliothèques. Ces documents offrent peut-

\footnotetext{
82 Le catalogue signale la Libri Bellarmini de scriptoribus ecclesiasticis continuatio, imprimée à Toul en 1665.
}

83 B.M. Nancy, ms. 441 (354). 
être une clé pour comprendre le rapport des religieux aux manuscrits médiévaux. Pour eux, le manuscrit est un objet moderne, chargé d'une certaine valeur affective parce qu'il a été produit par un frère, pour son usage tout en étant transmissible à la communauté. Du reste, alors que l'utilisation de ces manuscrits du quotidien est avérée, la consultation des manuscrits anciens est problématique: les religieux ne semblent pas les avoir beaucoup ouverts. Et pourquoi, du reste, ouvrir une Imitatio du XIVe siècle lorsqu'on dispose de plusieurs éditions récentes, en français, in octavo ou in duodecimo donc plus commodes à lire qu'un antique manuscrit ?84

Au cours du XVIIe siècle, le manuscrit médiéval connaît dans les bibliothèques conventuelles une évolution paradoxale. Quoique très minoritaire, il reste numériquement présent, y compris dans des couvents de fondation récente et dans le même temps, l'intérêt des religieux à l'égard de cet objet faiblit tandis que l'imprimé s'impose définitivement dans les collections. Celles-ci offrent aux laïcs une possible pérennisation des documents réunis au fil de toute une vie mais cette redistribution du patrimoine médiéval s'accompagne assez rarement d'une réelle curiosité pour la nature et le contenu de ces manuscrits. Au début du XVIII ${ }^{\text {e }}$ siècle encore, ce sont surtout les érudits laïcs ou réguliers (non mendiants) qui s'interrogent sur ces trésors alors qu'au sein des couvents, ces objets documentaires ne semblent pas faire l'objet d'un soin particulier. Ainsi, le bénédictin lorrain dom Calmet, rédigeant sa Bibliothèque lorraine, s'annonce au couvent des capucins de Toul où est décédé en 1720 le Père Benoît de Toul, éminent historien de Lorraine. Le bénédictin espère pouvoir consulter des notes rassemblées par le capucin et peut en effet lire quelques manuscrits de la fin du XV siècle, copies des œuvres de saint Jérôme effectuées par Didier Birstorff, chanoine et archidiacre de Toul, ancien précepteur du duc René II ${ }^{85}$. C'est au XVIIIe siècle seulement que les mendiants de province, bien après les bénédictins, les prémontrés ou les cisterciens, prendront la mesure de la spécificité ce support de l'écrit, au terme d'un processus de patrimonialisation ${ }^{86}$ qui fait apparaître à la conscience des religieux l'existence de corpus uniques dans leurs bibliothèques ${ }^{87}$.

\footnotetext{
${ }^{84}$ Le couvent possède alors deux éditions latines de l'Imitation (Lyon, 1570 et Paris, 1650) et une édition française (Paris, 1625).

${ }^{85}$ A. CALMET, Bibliothèque lorraine ou histoire des hommes illustres qui ont fleuri en Lorraine et dans les Trois-Evêchés, Nancy, 1751, col. 121.

${ }^{86}$ F. HENRYOT, «La patrimonialisation du manuscrit chez les réguliers au XVIIIe siècle », F. Henryot (dir.), L'historien face au manuscrit, du parchemin à la bibliothèque numérique, Louvain-la-Neuve, 2012, p. 127-145.

87 Voir par exemple le catalogue des grands carmes des Terreaux à Lyon (Arch. dép. Rhône, 11H41), rédigé sans doute à la fin du XVIIIe siècle, où une liste de « livres en velin manuscrits » est détaillée à la fin (quinze titres), tandis que des manuscrits plus ordinaires sont signalés dans les rubriques thématiques du catalogue, avec la simple mention « MC ».
} 